\title{
EMCCDs for Space Applications
}

\author{
David R. Smith ${ }^{* a}$, David M. Walton ${ }^{\text {b, Richard Ingley }}{ }^{\mathrm{a}}$, Andrew D. Holland ${ }^{\mathrm{a}}$, Mark Cropper ${ }^{\mathrm{b}}$, \\ Peter Pool ${ }^{\mathrm{c}}$ \\ a 2 v centre for electronic imaging, Brunel University, Uxbridge, Middlesex, UB8 3PH, UK; \\ ${ }^{\mathrm{b}}$ MSSL, Holmbury St. Mary, Dorking, Surrey, RH5 6NT, UK; \\ c 2 v technologies (uk) ltd, 106 Waterhouse Lane, Chelmsford, Essex, CM1 2QU, UK
}

\begin{abstract}
This paper describes a qualification programme for Electron-Multiplication Charge Coupled Devices (EMCCDs) for use in space applications. While the presented results are generally applicable, the programme was carried out in the context of CCD development for the Radial Velocity Spectrometer (RVS) instrument on the European Space Agency's cornerstone Gaia mission. We discuss the issues of device radiation tolerance, charge transfer efficiency at low signal levels and life time effects on the electron-multiplication gain. The development of EMCCD technology to allow operation at longer wavelengths using high resistivity silicon, and the cryogenic characterisation of EMCCDs are also described.
\end{abstract}

Keywords: EMCCD, X-ray detector, CTE, proton irradiation

\section{INTRODUCTION}

The Radial Velocity Spectrometer (RVS) on the European Space Agency's cornerstone Gaia mission is a photonstarved instrument where CCD resout noise is the dominant noise source. It is envisaged to use Electron-Multiplication Charge Coupled Devices (EMCCDs) to amplify the measured signal and improve the signal-to-noise ratio ${ }^{1-4}$. The operation of EMCCDs and their use in terrestrial applications has been well documented, but the technology has not, to date, been flown in space ${ }^{5,6}$. For the qualification of EMCCDs for space applications it was necessary to obtain a detailed understanding of the effects of radiation on device operation, in particular the effects of proton irradiation on the operation of the EMCCD gain register.

In the case of the EMCCDs for RVS, a development plan was put together that aimed to not only radiation qualify the EMCCD technology for space, but also to develop and test devices manufactured using higher resistivity silicon, required to improve the RVS quantum efficiency at longer optical wavelengths ${ }^{2,7,8}$. The first phase of the development plan involved the characterisation of two different commercially available EMCCDs from e2v technologies, the CCD65 and the CCD97. Both CCDs are frame transfer devices, manufactured using standard $20 \Omega$.cm silicon. Subsequent phases of the development plan involved the characterisation of CCD97 devices manufactured using higher resistivity silicon, to assess the effect of using high resistivity silicon on the electron-multiplication process and device charge transfer efficiency (CTE). The main device parameters of the different EMCCDs studied are given in Table 1. An overview of the development plan is shown in Figure 1, which also summarises some of the various tests carried out at each development phase.

This paper will first describe the electron-multiplication performance of the different EMCCDs tested and the development of the high resistivity CCD97 devices. The several radiation studies carried out to assess EMCCD radiation tolerance will then be presented, followed by a discussion of both CTE at low signal levels and life time effects on the electron-multiplication gain.

\footnotetext{
* David.Smith@brunel.ac.uk; phone +44 (0)189 526 6593; fax +44 (0)189 526 9773; www.brunel.ac.uk/cei
} 


\begin{tabular}{c|c|c|c}
\cline { 2 - 4 } & CCD65 & CCD97 & $\begin{array}{c}\text { CCD97 High } \\
\text { Resistivity }\end{array}$ \\
\hline Device type & Front illuminated & Back illuminated & Front illuminated \\
Operation & Frame Transfer & Frame Transfer & Frame Transfer \\
Active image area & $11.52 \times 8.64 \mathrm{~mm}$ & $8.192 \times 8.192 \mathrm{~mm}$ & $8.192 \times 8.192 \mathrm{~mm}$ \\
Image area active pixels & $288(\mathrm{H}) \times 576(\mathrm{~V})$ & $512(\mathrm{H}) \times 512(\mathrm{~V})$ & $512(\mathrm{H}) \times 512(\mathrm{~V})$ \\
Image pixel size & $20 \times 30 \mu \mathrm{m}$ & $16 \times 16 \mu \mathrm{m}$ & $16 \times 16 \mu \mathrm{m}$ \\
Gain register pixels & 591 & 526 & 526 \\
Resistivity & $20 \Omega . \mathrm{cm}$ & $20 \Omega . \mathrm{cm}$ & $100,1500 \Omega . \mathrm{cm}$ \\
\hline
\end{tabular}

Table 1: Parameters of the studied EMCCDs

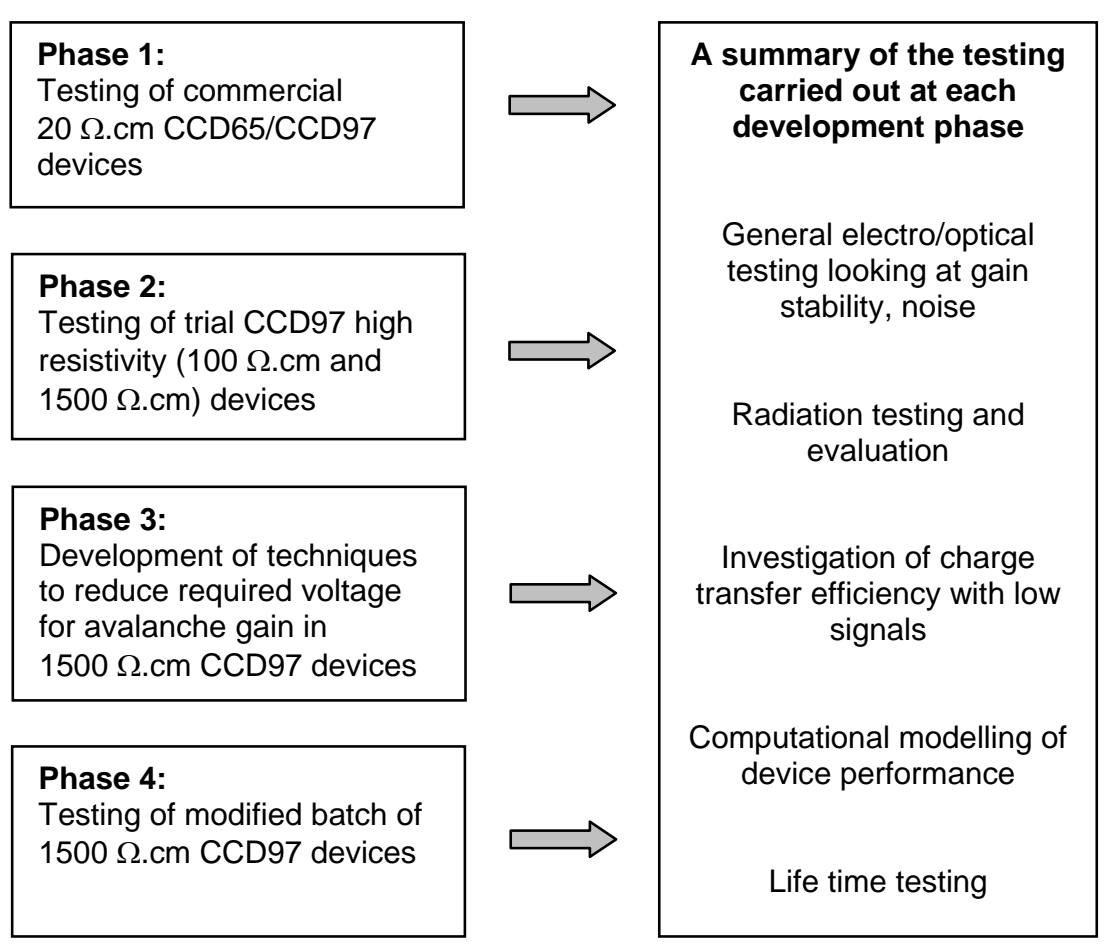

Figure 1: An overview of the RVS EMCCD development plan and the testing carried out during each phase

\section{EMCCD OPERATION FOR DIFFERENT TEMPERATURES AND RESISTIVITY}

The increase in signal-to-noise ratio achieved by an EMCCD is brought about by the inclusion of an additional 'multiplication' register following the standard readout register of the device. One electrode in each of the gain register pixels, $\mathrm{R} \phi \mathrm{HV}$, is pulsed to a much higher voltage than is needed to just transfer charge (typically $\sim 40-50$ volts). The electrode immediately before the high voltage electrode is held at $\sim 2$ volts d.c., generating a large electric field between 
the two electrodes which accelerates the charge carriers, causing impact ionisation to generate more electrons in the signal packet. After transfer through all the gain register pixels, gain of up to several hundred can be achieved, resulting in a greatly improved signal-to-noise ratio. The higher the voltage applied to $\mathrm{R} \phi \mathrm{HV}$, the greater the resulting gain in signal. For RVS, modest gains of only 8 to 10 are required to ensure the readout noise is below the background photon noise.

Devices were characterised using a vacuum chamber test facility incorporating a CryoTiger cooling system to enable testing from $+20{ }^{\circ} \mathrm{C}$ to $-130{ }^{\circ} \mathrm{C}$. The $\mathrm{CCD}$ and preamplifier headboard were mounted onto a copper cold finger attached to the CryoTiger cold head within the test chamber. Temperature control was provided by use of a Lakeshore controller (model number 331), using a PT-1000 resistor mounted on the copper cold finger in close proximity to the CCD. The temperature of the CCD could be maintained to within $\pm 0.05{ }^{\circ} \mathrm{C}$. An LED within the vacuum chamber was used to provide some optical signal to carry out CCD gain characterisation.

A second facility was also used for testing the electro-optical performance, in particular the CTE at low signal levels. In this facility, the CCD was mounted on a cold plate in a vacuum cryostat with an optical window. Cooling was provided by connection to a liquid nitrogen reservoir, with resistive heaters controlling the temperature. A modular optical bench was located outside the vacuum chamber, which allowed various illumination options from flat-field to single-pixel focussed images. The cold plate was mounted on a 2-D stepper motor stage to allow the CCD to be moved with respect to the illumination.

Figure 2 shows the gain achieved for a given R $\phi \mathrm{HV}$ voltage in a commercial $20 \Omega$.cm CCD97 device and the phase 2 initial trial batch of $100 \Omega . c m$ and $1500 \Omega . c m$ high resistivity CCD97 devices. In all presented data, the gain has been normalised to that with an $\mathrm{R} \phi \mathrm{HV}$ of 10 volts, where the gain is effectively unity so the device operates like a conventional CCD. The data were taken with the devices operating at $-100{ }^{\circ} \mathrm{C}$ in each case ${ }^{9}$.

The high resistivity devices were found to require a higher $\mathrm{R} \phi \mathrm{HV}$ voltage for a given gain than the standard $20 \Omega . \mathrm{cm}$ devices and also exhibited poor CTE at small signal levels (described in Section 5 below). These problems were rectified by $\mathrm{e} 2 \mathrm{v}$ technologies by a modification to the manufacturing process of the subsequent phase 3 batch of $1500 \Omega . c m$ high resistivity CCD97 devices. The improvement in the gain for a given R $\phi \mathrm{HV}$ in these modified devices can be seen in Figure 3, which compares the data taken from one of the modified $1500 \Omega . c m$ devices with the data from a $1500 \Omega . c m$ device in the first high resistivity batch ${ }^{10}$. The modification brings the gain achieved at a given R $\phi H V$ to a level comparable to that of the standard $20 \Omega . c m$ resistivity CCD97.

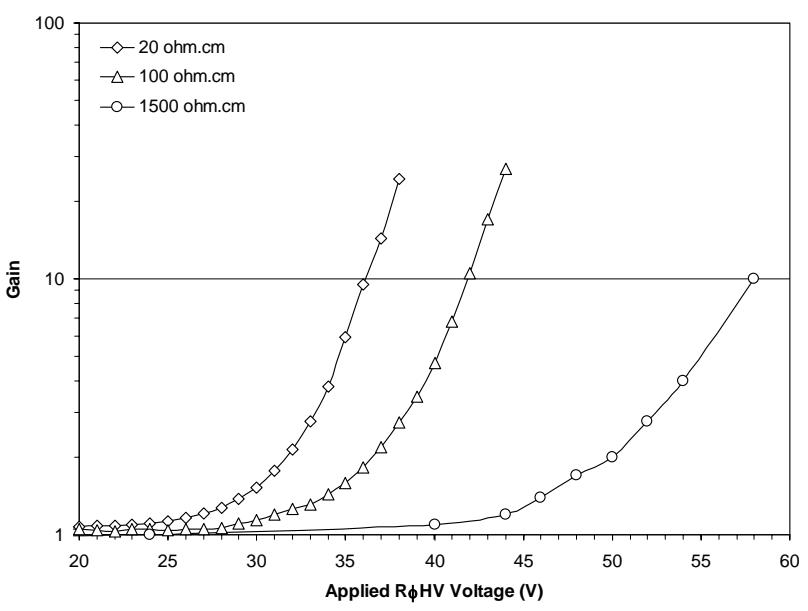

Figure 2: Gain vs. R $\phi \mathrm{HV}$ for commercial $20 \Omega . \mathrm{cm}$, and custom $100 \Omega . \mathrm{cm}$ and $1500 \Omega . c m$ CCD97 devices

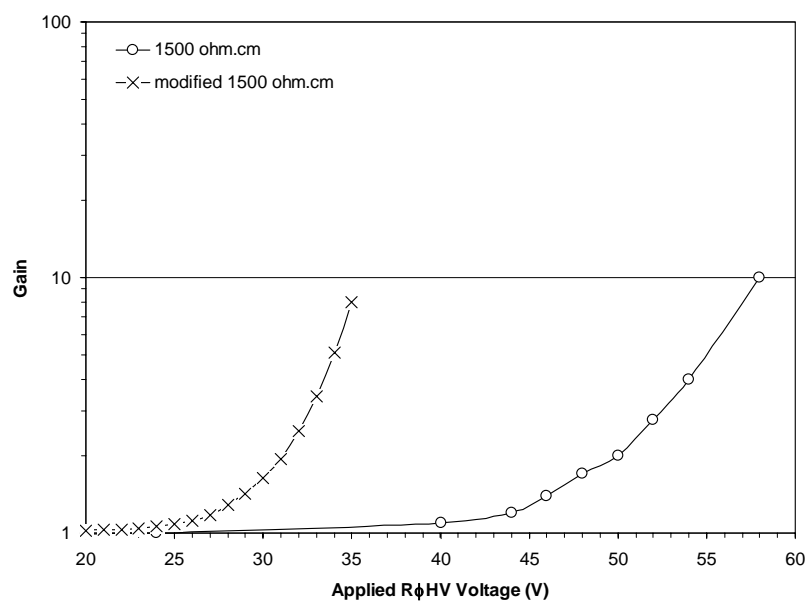

Figure 3: Gain vs. R $\phi \mathrm{HV}$ for the improved $1500 \Omega . c m$ CCD97 device 
Figure 4 shows the effect of temperature on the measured gain data from one of the modified $1500 \Omega . c m$ high resistivity CCD97 devices. The gain is seen to increase with decreasing temperature for a given R $\phi \mathrm{HV}$ voltage due to the temperature dependence of the electron ionisation rate. For a given electric field, the ionisation rate increases with decreasing temperature as described in detail by $\mathrm{Sze}^{11}$.

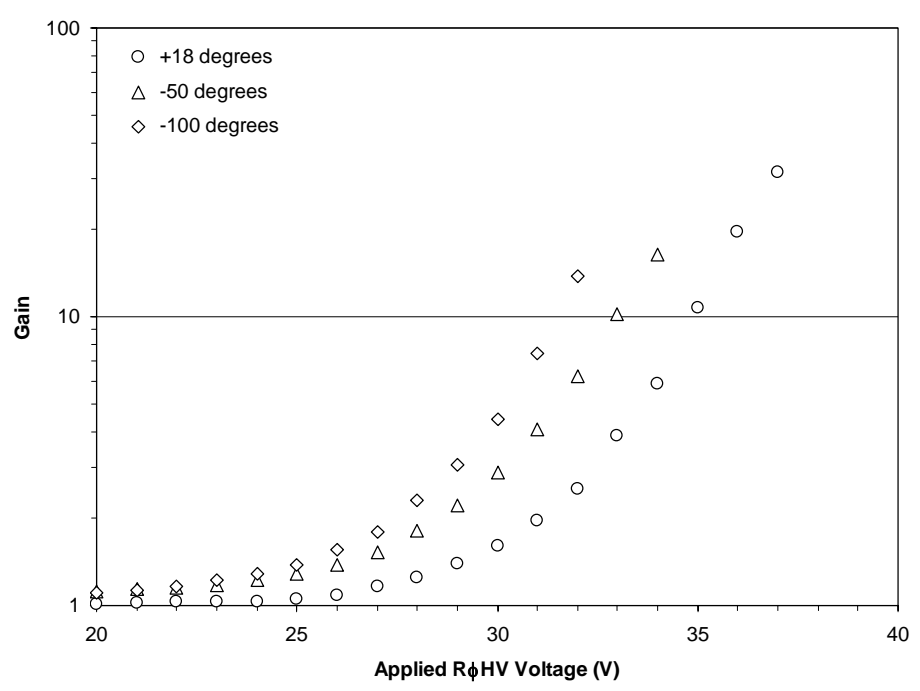

Figure 4: The effect of temperature on the recorded gain profile from an improved $1500 \Omega . c m$ CCD97 device

\section{RADIATION}

Prior to the acceptance of EMCCD technology in the CCDs of Gaia RVS, it was necessary to subject EMCCDs to radiation fluences representative of those expected to be received by the detectors during the 6 year Gaia mission. The Gaia spacecraft is to orbit the L2 point for the duration of its mission, and a detailed study of the radiation environment at this location was conducted by ESA to obtain estimates of the expected electron and proton fluences that would be accumulated by the RVS detectors. Depending on the point in the Solar cycle, this dose will be typical for other missions at L2. For silicon devices, the most important radiation component is that of protons, which can cause displacement damage in the silicon. The $10 \mathrm{MeV}$ equivalent Gaia RVS end-of-mission proton fluence is expected to be of the order of $\sim 2.5 \times 10^{9}$ protons. $\mathrm{cm}^{-2}$.

An EMCCD should behave in the same way as conventional CCDs in terms of operational changes resulting from radiation damage to the imaging area of the device, as there is no difference in construction. The generation of bright pixels and random telegraph signals caused by displacement damage, and any dark current increase or flat band voltage shifts caused by ionisation damage, will be the same as those observed in regular CCDs after irradiation, which are well described in the literature ${ }^{12-14}$. The main concern about using EMCCDs in the space radiation environment was the possibility of defect generation in the high field avalanche region of a gain register pixel, as a result of displacement damage. Such a defect could inject electrons into the pixel which, although at a low level, would then be amplified during transfer through the gain register. In the worst case, if such a defect was generated near the input end of the gain register, the injected charge could be subject to a gain $\sim 1,000$ before reaching the CCD output amplifier, saturating the recorded CCD image and possibly even causing catastrophic device failure. Other possibilities were that the defect would simply increase the gain in a given region of the gain register following the bright pixel, or that there would be no significant effect on the gain register operation at all after radiation damage, so that the resulting radiation damage effects would be the same as those in the readout register of a conventional CCD. 
In order to address the radiation concerns, a series of proton irradiation studies were carried out to assess the radiation tolerance of EMCCDs. There is a limit to the level of proton irradiation a given device can be subjected to and still remain in a state that can provide useful performance measurements. It was therefore necessary to carry out irradiation of a large number of devices in order to obtain good statistics on the possibility of device failure. To begin with, two CCD65 devices were irradiated with protons before subsequent irradiation studies were carried out, involving higher numbers of devices and increased proton fluence. Table 2 lists the four proton irradiation campaigns carried out during 2003-2005 and details the device type, the number of devices involved and $10 \mathrm{MeV}$ equivalent proton fluence in each case. Proton irradiations were carried out using particle accelerator facilities at the Paul Scherrer Institut in Switzerland and also at Birmingham University in the UK. The error associated with the dosimetry in each case was estimated to be $\sim 20 \%$ by the accelerator staff. All irradiations were carried out with the devices un-biased and at room temperature.

\begin{tabular}{|c|c|c|c|c|c|}
\hline $\begin{array}{l}\text { Irradiation } \\
\text { Campaign }\end{array}$ & Device Type & $\begin{array}{c}\text { Number of } \\
\text { Devices }\end{array}$ & \multicolumn{2}{|c|}{$\begin{array}{l}\text { Resistivity, Front or } \\
\text { Back Illumination }\end{array}$} & $\begin{array}{l}10 \text { MeV Equivalent } \\
\text { proton fluence } \\
\text { (protons.cm }{ }^{-2} \text { ) }\end{array}$ \\
\hline 1 & $\begin{array}{l}\text { CCD65 } \\
\text { CCD65 }\end{array}$ & $\begin{array}{l}1 \\
1\end{array}$ & $\begin{array}{l}20 \Omega . \mathrm{cm} \\
20 \Omega . \mathrm{cm}\end{array}$ & $\begin{array}{l}\mathrm{FI} \\
\mathrm{FI}\end{array}$ & $\begin{array}{l}5 \times 10^{8} \\
2 \times 10^{9}\end{array}$ \\
\hline 2 & CCD65 & 20 & $20 \Omega . c m$ & $\mathrm{FI}$ & $2.5 \times 10^{9}$ \\
\hline $3^{*}$ & CCD65 & 8 & $20 \Omega . c m$ & $\mathrm{FI}$ & $2 \times 10^{10}$ \\
\hline 4 & $\begin{array}{l}\text { CCD97 } \\
\text { CCD97 } \\
\text { CCD97 }\end{array}$ & $\begin{array}{c}94 \\
1 \\
1\end{array}$ & $\begin{array}{l}20 \Omega . \mathrm{cm} \\
100 \Omega . \mathrm{cm} \\
1500 \Omega . \mathrm{cm}\end{array}$ & $\begin{array}{l}\mathrm{BI} \\
\mathrm{FI} \\
\mathrm{FI}\end{array}$ & $\begin{array}{l}2 \times 10^{10} \\
2 \times 10^{10} \\
2 \times 10^{10}\end{array}$ \\
\hline
\end{tabular}

* this irradiation campaign used 8 of the devices irradiated in campaign 2 , raising their total proton fluence received to that given in the table

Table 2: EMCCD irradiation campaigns

As the principal area of concern in the radiation studies was the gain register, for the CCD65 testing, a method of looking only at the charge distribution through the readout and gain registers in a device was utilised. Figure 5 shows a cartoon of the obtained charge profile which would be acquired by clocking the whole gain register and readout register after an integration time which is significantly shorter than the readout time, preventing thermal leakage current from the image and store sections of the device entering the readout register. Moving from left to right in the cartoon profile, the first 16 pixels are lead-in pixels (underscan), and exhibit no gain. Following these pixels are the 591 gain register pixels. The small step increase in dark current level from the last lead-in pixel to the first gain register pixel is due to the larger physical size of the gain register pixels that allow them to hold the increased signal charge. The subsequent rise in dark current through the pixels of the gain register is a result of each charge packet having passed through one more gain register pixel than its predecessor, the final gain register pixel having a gain associated with transfer through the entire gain register. The subsequent flat portion of the trace is the dark current level in the pixels of the standard readout register in the device, all of which have had their dark current amplified by passage through the entire gain register, and hence have a higher signal than the lead-in pixels. The step downward from the peak signal in the gain register at the beginning of this section is due to the readout register pixels having smaller physical area than the gain register pixels. Finally, there are some overscan pixels, which have very little accumulated dark current (because they accumulate dark current only during the readout), but have all been through the entire gain register. 


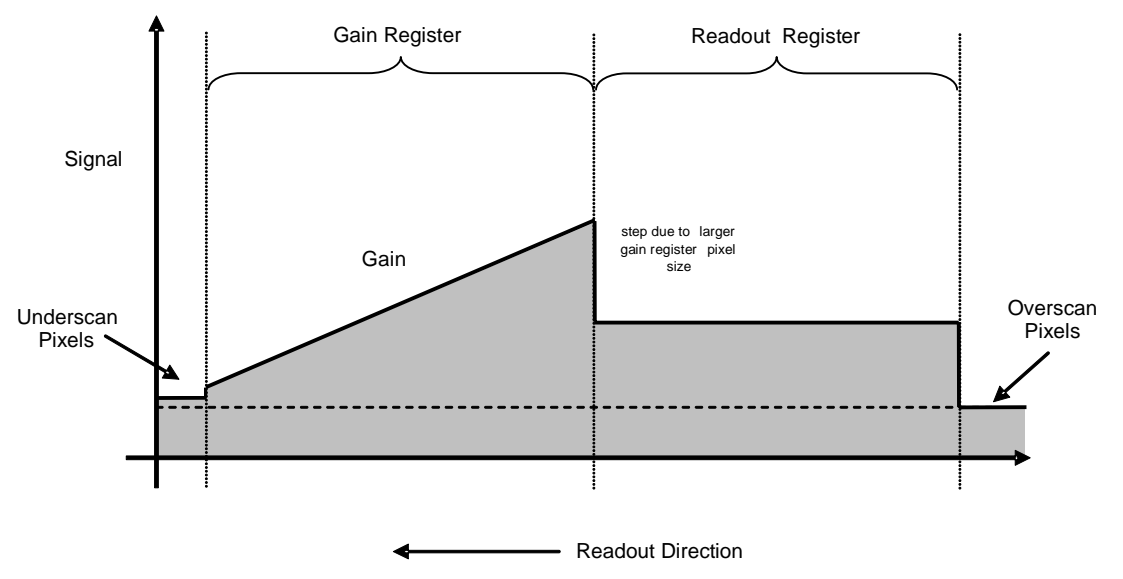

Figure 5: A cartoon of the expected readout and gain register signal profile

The first campaign involved two CCD65 devices (Table 2), one irradiated to a fluence level comparable to the predicated end of mission proton fluence for XMM-Newton, the second irradiated to a level representative of the expected Gaia end of mission fluence. In each case, both devices were found to be fully functional after irradiation. After this initial step in characterising the radiation tolerance of EMCCDs, the second irradiation campaign incorporated 20 CCD65 devices to improve statistics on possible device failure. Again, all devices were found to operate after irradiation to the expected Gaia end of mission proton fluence, prompting the next irradiation campaign to increase the proton fluence by an order of magnitude.

The third irradiation campaign increased the proton dose given to 8 of the CCD65 devices to $2 \times 10^{10}$ protons. $\mathrm{cm}^{-2}$, a factor 10 times the expected Gaia RVS end of mission dose. Figures 6 and 7 respectively show recorded charge profiles from an unirradiated CCD65 and a CCD65 irradiated to a fluence of $2 \times 10^{10}$ protons. $\mathrm{cm}^{-2}$ in the third irradiation campaign. In each figure the same three $\mathrm{R} \phi \mathrm{HV}$ voltages were used in each case, highlighting the increase in dark current level caused by radiation damage. (Dark current is however negligible at operating temperatures of $-100{ }^{\circ} \mathrm{C}$.) Bright pixel charge spikes are visible in the gain register in the profiles of Figure 7, but they have not caused any steps to occur in the gain slope portion of the curve. These bright pixels are also not significantly amplified when R $\phi \mathrm{HV}$ is increased. This indicates that the defects have not caused any significant change to the device operation, the amount of gain for a given $\mathrm{R} \phi \mathrm{HV}$ being comparable to that prior to irradiation.

After all three CCD65 radiation studies, all 22 devices tested were found to be fully functional with no catastrophic failures, and the radiation damage induced dark current and bright pixel generation levels were comparable from device to device for a given proton fluence and comparable to the results of irradiation studies using conventional CCDs. In terms of Gaia RVS, for which the CCDs under development have a similar gain register length, the third irradiation campaign study was equivalent to testing 5 complete RVS focal planes to the expected end of mission proton fluence, indicating that the risk of catastrophic failure of any devices is low.

In order to further improve statistics on the possibility of EMCCD device failure due to radiation damage, a further study involving 100 CCD97 was carried out in 2005. Of these devices, 6 were held as controls while 94 were irradiated to a fluence of $2 \times 10^{10}$ protons. $\mathrm{cm}^{-2}$, a test amounting to the irradiation of an RVS focal plane to several tens of times the expected mission dose. The outcome of this study was again very positive, with all 94 devices operating after irradiation within expected parameters. Figure 8 shows the average recorded gain variation with $\mathrm{R} \phi \mathrm{HV}$ of the devices 
both before and after irradiation at $+18{ }^{\circ} \mathrm{C}$. There is no significant change in the recorded profile after irradiation, indicating that the gain function of an EMCCD is unaltered by proton damage. It should be noted that the gain curve after irradiation does not extend as far to the right in the plot, due to the increased dark current from radiation damage saturating the CCD output amplifier above a gain of $\sim 6$. One of each type of high resistivity CCD97 device, $100 \Omega . c m$ and $1500 \Omega . \mathrm{cm}$, were also irradiated during the fourth campaign, the recorded gain profiles from each device after irradiation also show no significant deviation from that recorded prior to irradiation. Figure 9 shows the measured gain profiles before and after irradiation from the irradiated $100 \Omega . \mathrm{cm}$ high resistivity device. Unfortunately data at $-100{ }^{\circ} \mathrm{C}$ could not be obtained prior to the irradiation of the device, the pre-irradiation data being recorded with the device operating at $+18{ }^{\circ} \mathrm{C}$, but the shift in the gain profile as a result of the temperature difference between the data sets is comparable to the temperature variation observed in test results from other EMCCD devices studied (c.f. Figure 4). The various radiation studies and results are discussed in more detail in the literature ${ }^{15-17}$.

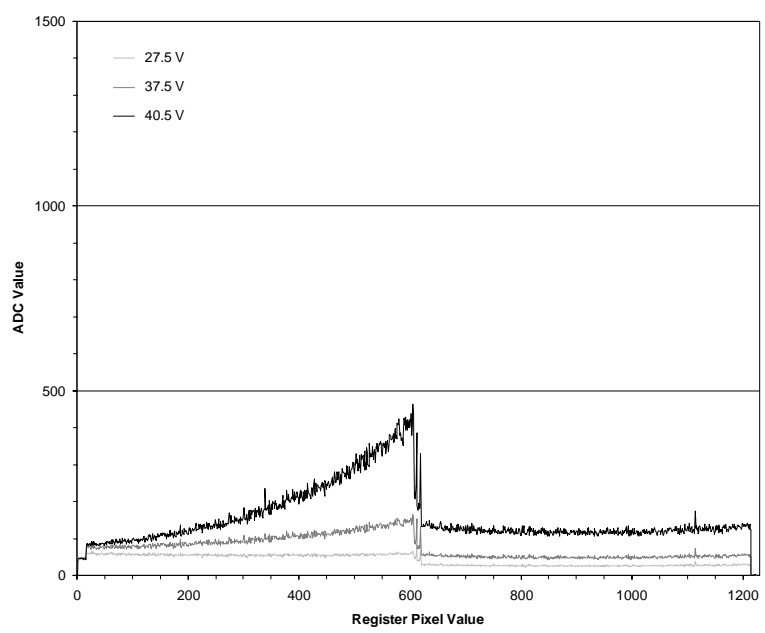

Figure 6: Readout charge profiles from an unirradiated CCD65 at three different $\mathrm{R} \phi \mathrm{HV}$ voltages

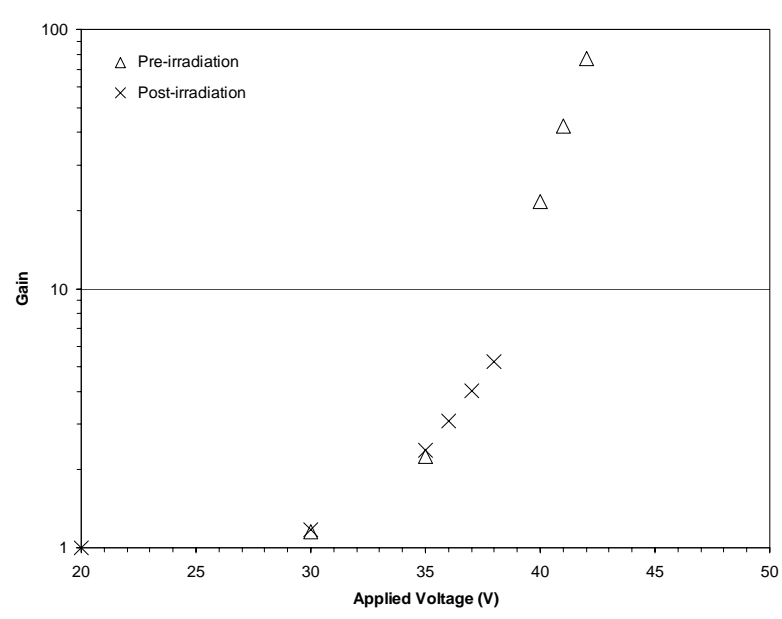

Figure 8: The average recorded gain profile from the irradiated CCD97 devices before and after irradiation to $2 \times 10^{10}$ protons. $\mathrm{cm}^{-2}$

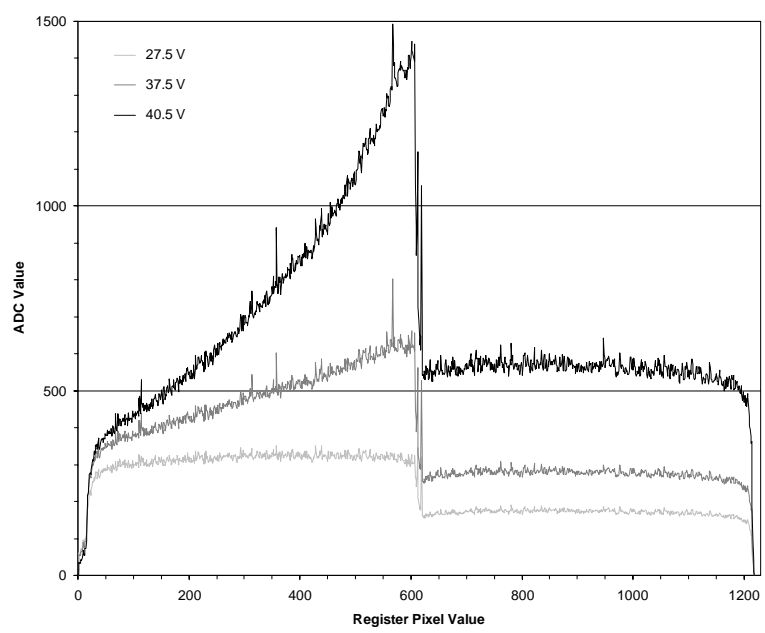

Figure 7: Readout charge profiles from a CCD65 irradiated to $2 \times 10^{10}$ protons. $\mathrm{cm}^{-2}$, at three different $\mathrm{R} \phi \mathrm{HV}$ voltages

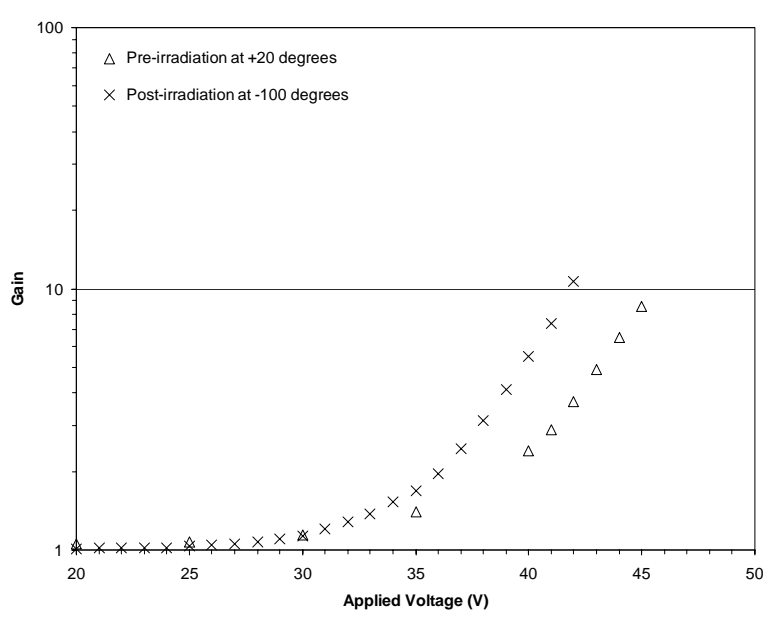

Figure 9: Recorded gain profiles from a $100 \Omega . c m$ high resistivity device, both before and after irradiation to $2 \times 10^{10}$ protons. $\mathrm{cm}^{-2}$ 
To assess the significance of the radiation damage results, a discussion of the damage expectation is useful. The volume of the high field region in a given gain register pixel is not clear, but it can be estimated to have a depth of $0.5 \mu \mathrm{m}$ over a nominal area of $20 \mu^{2}$. Analysis has shown that silicon displacements occur at $\sim 0.01$ displacements $/$ proton $/ \mu \mathrm{m}$ of track length. Of these displacements $\sim 98 \%$ re-combine, leaving an estimated $2 \times 10^{-4}$ displacements $/$ proton $/ \mu \mathrm{m}$. A proton fluence of $2 \times 10^{10}$ protons. $\mathrm{cm}^{-2}$ will therefore produce $\sim 0.4$ damage sites within the high field region of each gain register pixel ${ }^{18}$. Given there are 526 gain register pixels in a CCD97, the irradiation of 94 CCD97 devices should result in $\sim 20,000$ damage sites in the vicinity of the high field region in each gain register pixel. If this is the case, $\sim 2,000$ of these damage sites will lie within the first $10 \%$ of the gain register, where their presence would be most noticeable. This estimate is subject to great error, but even with an error of a factor of 100, defects should have been seen in several devices. The absence of any significant proton damage effects in the gain register of all the 118 EMCCDs irradiated in the presented studies, 104 of which were irradiated to 10 times the Gaia mission fluence, is very encouraging.

A separate radiation study was carried out in 2003 to assess the impact of ionising $\mathrm{Co}^{60}$ irradiation on EMCCD device performance $^{19}$. This study found that no device failure or unexpected operating characteristics in the EMCCDs tested occurred as a result of $\mathrm{Co}^{60}$ gamma irradiation.

\section{GAIN STABILITY AND LIFE TESTING}

Another important issue for the qualification of EMCCDs for space applications was an assessment of the device avalanche multiplication gain stability over long periods of time. To address this issue, two of the modified $1500 \Omega . \mathrm{cm}$ CCD97 high resistivity devices were utilised as part of a life testing programme. The two devices were provided in an 'unconditioned' state. The standard practice of EMCCD conditioning (operation with high gain for an extended time period) is usually carried out by $\mathrm{e} 2 \mathrm{v}$ technologies to stabilise the gain variation at a given R $\phi \mathrm{HV}$ over time. The 'virgin' state of the devices allowed the initial drop in gain for a given $\mathrm{R} \phi \mathrm{HV}$ in the first few days of operation to be observed as part of the life testing study.

Life time gain stabilisation data had previously been obtained for EMCCDs operating with a gain of 1000 at TV-rate readout. Figure 10 shows the variation in $\mathrm{R} \phi \mathrm{HV}$ for a gain of 1000 in an EMCCD operating at room temperature. After the initial conditioning period of $\sim 100$ hours, the gain becomes much more stable, with an R $\phi \mathrm{HV}$ voltage increase of $\sim 2 \mathrm{~V}$ over a period of $\sim 17,000$ hours being required to maintain a gain of 1000 . For this study, life time gain stability data from the new high resistivity CCD97 devices was required at slower readout speed, lower operating temperature and lower gain. The life testing of the two CCD97 devices was carried out at an operating temperature of $-100{ }^{\circ} \mathrm{C}$, for gain values of 6, 8 and 10 (the specified gain for Gaia RVS is 8).

Life testing was carried out using the same vacuum chamber facility as described in Section 2 above. The same clock voltages and bias voltages were used for each device and, while not being tested, the applied R $\phi \mathrm{HV}$ voltage was held at $40 \mathrm{~V}$. It should be noted that this is a few volts higher than that required for the specified RVS gain of 8 , around 35 volts, so as to slightly stress the devices. While data collection was not taking place, the LED used to provide some charge signal for gain calibration was switched off. As of January 2006, the two life test devices had undergone 1300 hours of continuous operation ${ }^{20}$. During each data collection period, three gain profiles were obtained in succession, to assess the repeatability of the measurements. Figure 11 shows three such gain profiles measured after 1300 hours of life testing, the repeatability of the measurements during any given data taking session lying within $10 \%$.

An initial sharp increase in required $\mathrm{R} \phi \mathrm{HV}$ for a given gain was observed, occurring in the first few days, a time comparable to that shown in Figure 10. The measured gain profiles were then seen to stabilise to within $\sim 0.2 \mathrm{~V}$ over the remaining duration of the life testing to date. Figure 12 shows the recorded variation in $\mathrm{R} \phi \mathrm{HV}$ for gain values of 6,8 and 10 in one of the two tested devices. 


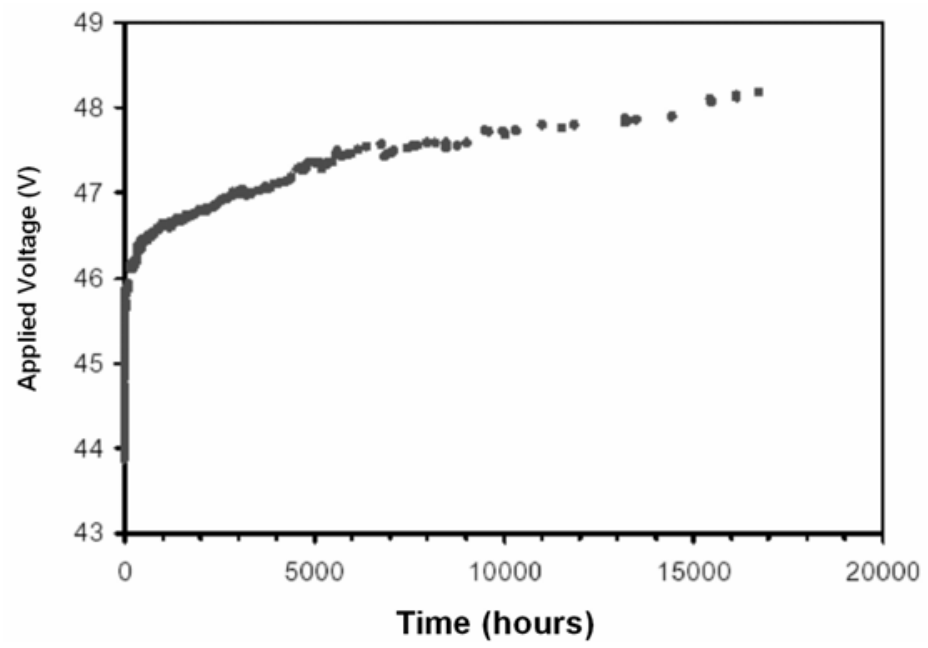

Figure 10: Variation in required $\mathrm{R} \phi \mathrm{HV}$ for a gain of 1000 with operational life time, for an EMCCD operating at room temperature

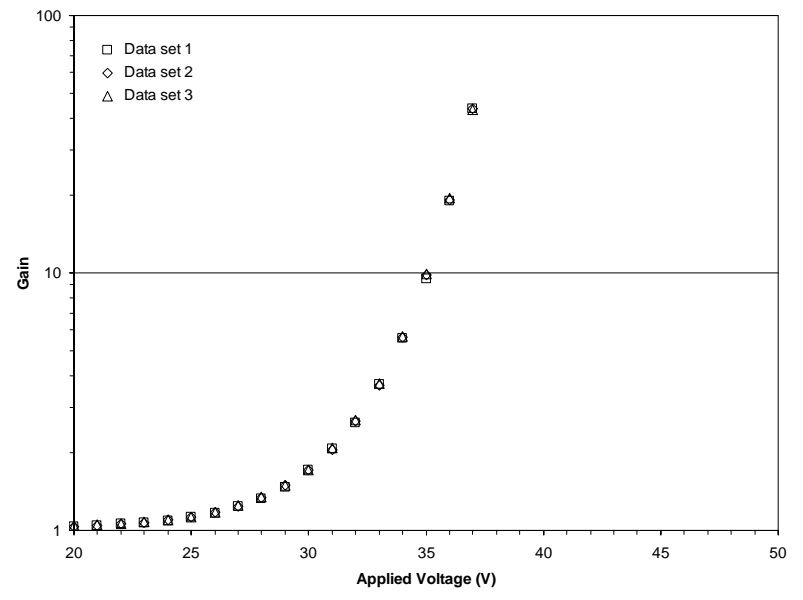

Figure 11: Recorded gain profiles from a modified $1500 \Omega . c m$ CCD97 operating at $-100{ }^{\circ} \mathrm{C}$ after 1300 hours of life testing

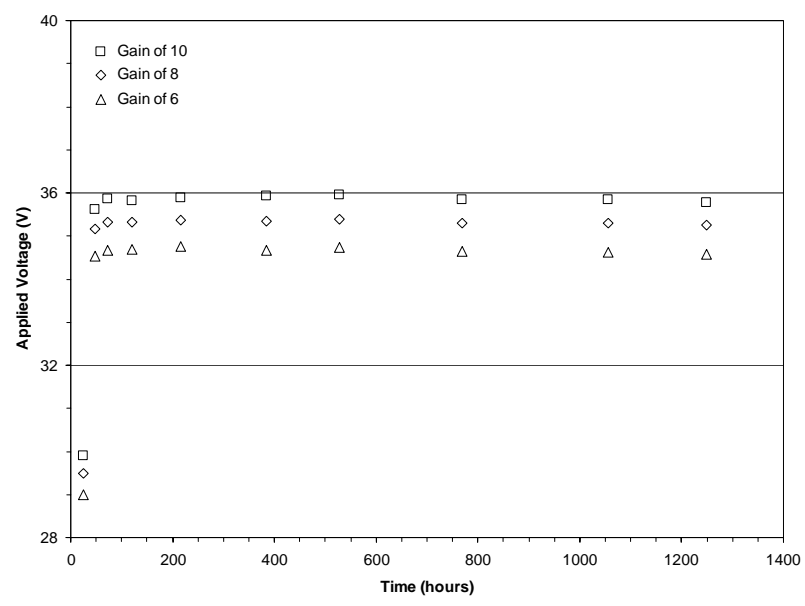

Figure 12: Required $\mathrm{R} \phi \mathrm{HV}$ for gain values of 6,8 and 10 in a modified $1500 \Omega$.cm CCD97 operating at $-100{ }^{\circ} \mathrm{C}$

\section{CHARGE TRANSFER EFFICIENCY}

A key performance parameter investigated as part of the RVS CCD development plan was the CTE in the gain register for very low light levels in the high resistivity CCD97 devices. Good CTE at $\sim$ single electron signal levels has been demonstrated for conventional $20 \Omega$.cm EMCCDs and for classical CCDs but CTE measurements from high resistivity EMCCDs had not been previously recorded. 
Figure 13 shows three test images obtained from one of the initial batch of $1500 \Omega . c m$ CCD97 devices, operating at $-100{ }^{\circ} \mathrm{C}$ with a photoelectron signal level of a few hundred electrons. Operating at a gain of 1 , the serial CTE (the horizontal direction in the presented CCD images) is good. However, the charge transfer through the L3 register becomes worse as gain is increased, which is evident from the increased horizontal blurring in the subsequent images recorded at a gain of 5 and 10 respectively. It should be noted that the vertical streaking in the images is not a device defect, but is a result of frame-shift smear caused by the particular clocking sequence used to read out the device.

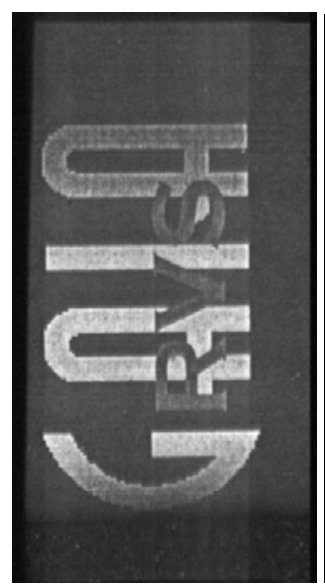

Gain = 1

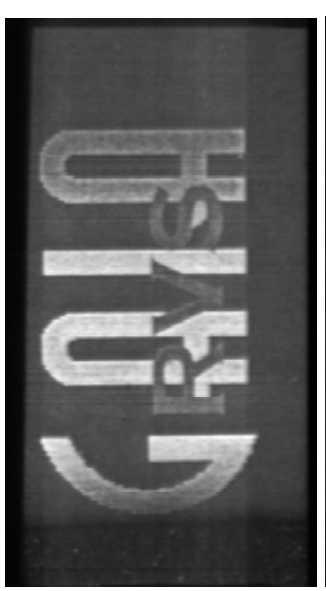

Gain $=5$

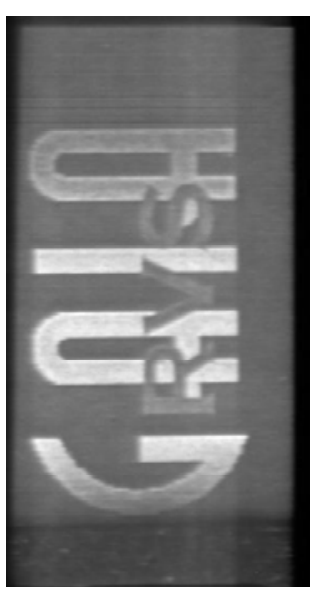

Gain $=10$

Figure 13: Images from a $1500 \Omega . c m$ EMCCD device, showing the increase in serial CTE as gain is increased

Figure 14 shows images acquired of a pinhole optical source, imaged on to approximately a single CCD pixel for one of the $100 \Omega$.cm devices, again operating at $-100^{\circ} \mathrm{C}$. The image on the left is a single exposure of $\sim 100$ photoelectrons operating with a gain of 10 , while the image on the right is a composite of 1000 image frames, with an average of $\sim 1$ photoelectron per frame, operating at the same gain. The images show that the EMCCDs manufactured using silicon of $100 \Omega . \mathrm{cm}$ have good serial CTE (the horizontal direction in the presented CCD images) at single photoelectron levels. However, at much higher gain, when $\mathrm{R} \phi \mathrm{HV}$ is increased beyond $\sim 50 \mathrm{~V}$ (giving a gain of $\sim 650$ ) the gain register CTE does begins to degrade. Similar behaviour was observed in the initial batch of $1500 \Omega . \mathrm{cm}$ devices, where the effect was more apparent at lower gain as a result of the lower gain achieved for a given voltage. A signal dependence of the CTE was also observed during the testing. For example, at a gain of 10, the serial CTE was acceptable for signal levels above $\sim 1000$ photoelectrons, but as signal levels are reduced, smearing in the gain register starts to be seen.

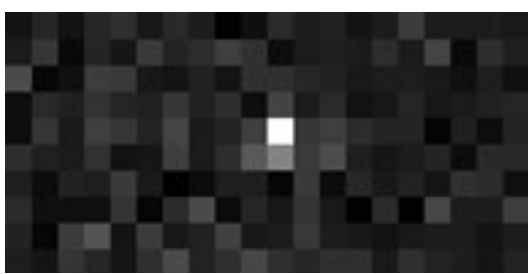

single frame of $\sim 100$ photoelectrons

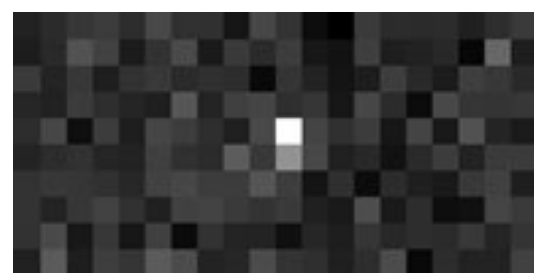

1000 frames at an average level of $\sim 1$ photoelectron

Figure 14: Images from a $100 \Omega . c m$ EMCCD operating with a gain of 10, showing good serial CTE at single photoelectron signal levels 
The CTE was also investigated in the two irradiated high resistivity devices. In the same way that radiation effects influence the CTE of conventional devices, it was found to be necessary to include a pre-flash charge signal into the image array preceding the pinhole source to fill radiation induced trapping sites. Table 3 lists the percentage of a 25 photoelectron signal retained in the central pixel for varying pre-flash signal sizes. The data shown are for the $100 \Omega . \mathrm{cm}$ device after irradiation to $2 \times 10^{10}$ protons. $\mathrm{cm}^{-2}$. The observed charge loss resulting from radiation damage is consistent with the Monte Carlo modelling study carried out as part of the RVS CCD development plan which demonstrated that pre-flash, or use of charge injection, was required to regain CTE after radiation damage ${ }^{21}$. This effect is not specific to EMCCDs and is observed in conventional CCDs. Note that the CTE degradation occurred in the parallel clocking direction rather than the serial direction, showing that CTE in the gain register was not significantly degraded.

\begin{tabular}{c|c}
\hline Pre-flash signal (e) & Retained signal (\%) \\
\hline 2400 & 100 \\
\hline 600 & 92 \\
\hline 150 & 40 \\
\hline 75 & 20 \\
\hline 35 & 0 \\
\hline
\end{tabular}

Table 3: The amount of a 25 electron signal retained for a given pre-flash charge signal

\section{CONCLUSION}

The paper has given an overview of the many EMCCD studies that have taken place since 2001 as part of the Gaia RVS CCD development plan, to qualify electron-multiplication CCD technology for application in space. A major issue for concern in using EMCCDs in the space environment was the radiation damage susceptibility of the gain register. In particular, it was necessary to investigate the possibility that radiation damage to the high field regions within the gain register pixels of the device could cause severe operational problems and even device failure. After a series of radiation studies on increasingly large numbers of devices and with proton fluences an order of magnitude larger than that expected in a typical space mission at L2, no degradation in operational performance beyond that which would be expected in a classical CCD has been observed, and the concern about the irradiation of the gain register in EMCCDs has been found mitigated. The testing of 94 EMCCDs to a $10 \mathrm{MeV}$ equivalent fluence of $2 \times 10^{10}$ protons.cm $^{-2}$ is equivalent to testing 50 complete Gaia RVS focal planes to the expected Gaia end of mission proton fluence and resulted in no device failures, the characteristic gain profile of each device showing no significant change after irradiation.

Further work addressed the development of high resistivity EMCCDs. These devices are found to exhibit no unexpected radiation damage effects, in line with the irradiation testing of conventional $20 \Omega$.cm EMCCDs. The parallel and serial CTE for small signal sizes has been shown to be good. The effect of radiation on the parallel and serial CTE in EMCCDs is comparable to that in conventional devices, the use of charge injection of pre-flash being a suitable technique to regain CTE after irradiation.

This work has greatly improved confidence that EMCCDs can be used in space with a very low risk of catastrophic failure, and a number of future space applications of EMCCDs have been proposed, including Gaia RVS, ground scanning applications and LIDAR ${ }^{22}$. 


\section{ACKNOWLEDGMENTS}

The authors would like to thank e2v technologies (uk) ltd for supplying the EMCCD devices used in the various studies presented in this paper, ESA and PPARC for providing funding for the proton irradiation studies, and the staff at PSI and Birmingham University for helping with the irradiation programmes.

\section{REFERENCES}

1. M. Cropper, K. Mason, MSC-RVS-001, $10^{\text {th }}$ July 2001, ESA Livelink.

2. M. Cropper, D. Walton, A. Holland, D. Smith, D. Katz, "CCD Detector for Gaia-RVS", MSSL/GaiaRVS/TN/017.02, $16^{\text {th }}$ October 2005, ESA Livelink.

3. A. D. Holland, M. Cropper, D. Katz, I. B. Hutchinson, R. M. Ambrosi, T. Stevenson, D. R. Smith, D. Walton, D. Burt, P. Pool, D. Morris, T. Paulsen, "CCDs for the Radial Velocity Spectrometer on GAIA", Proc. SPIE, vol. 5251, (2004), pp. 253-60.

4. A. D. Holland, M. A. Perryman, C. Vetel, R. Steward, I. B. Hutchinson, R. M. Ambrosi, D. R. Smith, S. Bowring, D. J. Burt, T. Eaton, D. G. Morris, P. Pool, R. Davancens, A. Laborie, P. Pouny, F. Safa, I. Zayer, A. D. Short, "Development of the CCDs for ESA's GAIA cornerstone mission", Proc. SPIE, vol. 5167, (2004), pp. 38-49.

5. P. Jerram, P. Pool, R. Bell, D. Burt, S. Bowring, S. Spencer, M. Hazelwood, I. Moody, N. Catlett, P. Heyes, "The LLLCCD: Low light imaging without the need for an intensifier," Proc. SPIE, vol. 4306, 2001.

6. C. D. Mackay, R. N. Tubbs, R. Bell, D. Burt, P. Jerram, I. Moody, "Sub-electron read noise at MHz pixel rates," Proc. SPIE, vol. 4306, 2001, pp. 289-98.

7. M. Cropper, A. Holland, D. Walton, "CCD Development Plan for Gaia-RVS", MSSL/Gaia-RVS/PP/001.02, $20^{\text {th }}$ July 2004, ESA Livelink.

8. M. Cropper, A. Holland, D. Walton, "L3CCD Radiation Results: Implications for RVS", MSSL/GaiaRVS/TN/012.01, $24^{\text {th }}$ June 2004, ESA Livelink.

9. D. Smith, R. Ingley, A. Holland, "e2v technologies High Resistivity L3Vision ${ }^{\mathrm{TM}}$ Characterisation", BRUNEL/GAIA-RVS/TN/023.01, $15^{\text {th }}$ July 2005, ESA Livelink.

10. D. Smith, A. Holland, R. Ingley, "Characterisation of improved $1500 \Omega \mathrm{cm}$ L3Vision CCDs", BRUNEL/GAIARVS/TN/026.01, $21^{\text {st }}$ October 2005, ESA Livelink.

11. Sze, S. M., "Physics of Semiconductor Devices", John Wiley and Sons Ltd., 2nd Ed., 1981.

12. Holmes-Siedle, A., L. Adams, "Handbook of Radiation Effects", Oxford University Press, 2nd Ed., 2002.

13. D. R. Smith, A. D. Holland, M. S. Robbins, R. M. Ambrosi, I. B. Hutchinson, "Proton induced leakage current in CCDs", Proc. SPIE, vol. 4851, (2003), pp. 842-48.

14. D. R. Smith, A. D. Holland, I. B. Hutchinson, "Random Telegraph Signals in Charge Coupled Devices", Nuc. Inst. Meth., vol. A530, (2004), pp. 521-35.

15. D. R. Smith, A. D. Holland, M. S. Robbins, "The effect of protons on E2V Technologies L3Vision CCDs", Nuc. Inst. Meth., vol. A513, (2003), pp. 296-99.

16. D. R. Smith, A. D. Holland, "Proton irradiation of e2v technologies L3Vision devices", Nuc. Inst. Meth., vol. A546, (2005), pp. 544-52.

17. D. R. Smith, R. Ingley, A. D. Holland, "Proton irradiation of EMCCDs", IEEE Trans. Electron Devices, vol. 53, no. 2, (2006), pp. 205-10.

18. P. Poole, e2v technologies ltd., private communication, $1^{\text {st }}$ June 2004.

19. B. Hadwen, M. Camas, M. Robbins, "The effects of $\mathrm{Co}^{60}$ Gamma Radiation on Electron Multiplying Charge Coupled Devices", Proc. RADECS, (2003).

20. R. Ingley, D. R. Smith, A. D. Holland, C. Castelli, "L3CCD Life Testing”, BRUNEL/GAIA-RVS/TN/027.02, $12^{\text {th }}$ December 2005, ESA Livelink.

21. I. Hutchinson, D. Smith, A. Holland, “Gaia RVS Simulation”, BRUNEL/GAIA-RVS/TN/025.02, $8^{\text {th }}$ September 2005, ESA Livelink.

22. P. J. Pool, D. G. Morris, D. J. Burt, R. T. Bell, A. D. Holland, D. R. Smith, "Application of electron multiplying CCD technology in space instrumentation", Proc. SPIE, vol. 5902, (2005). 